\title{
Cytokinins Act Synergistically with Salicylic Acid to Activate Defense Gene Expression in Rice
}

\author{
Chang-Jie Jiang, ${ }^{1}$ Masaki Shimono, ${ }^{1}$ Shoji Sugano, ${ }^{1}$ Mikiko Kojima, ${ }^{2}$ Xinqiong Liu, ${ }^{1}$ Haruhiko Inoue, ${ }^{1}$ \\ Hitoshi Sakakibara, ${ }^{2}$ and Hiroshi Takatsuji ${ }^{1}$ \\ ${ }^{1}$ Disease Resistant Crops Research Unit, National Institute of Agrobiological Sciences, Kannondai 2-1-2, Tsukuba, 305- \\ 8602 Japan; ${ }^{2}$ Plant Productivity Systems Research Group, RIKEN Plant Science Center, Suehiro 1-7-22, Tsurumi, \\ Yokohama 230-0045, Japan
}

Submitted 13 June 2012. Accepted 4 October 2012.

\begin{abstract}
Hormone crosstalk is pivotal in plant-pathogen interactions. Here, we report on the accumulation of cytokinins (CK) in rice seedlings after infection of blast fungus Magnaporthe oryzae and its potential significance in rice$M$. oryzae interaction. Blast infection to rice seedlings increased levels of $N^{6}$-( $\Delta^{2}$-isopentenyl) adenine (iP), iP riboside (iPR), and iPR 5'-phosphates (iPRP) in leaf blades. Consistent with this, CK signaling was activated around the infection sites, as shown by histochemical staining for $\beta$-glucuronidase activity driven by a CK-responsive OsRR6 promoter. Diverse CK species were also detected in the hyphae (mycelium), conidia, and culture filtrates of blast fungus, indicating that $M$. oryzae is capable of production as well as hyphal secretion of CK. Co-treatment of leaf blades with CK and salicylic acid (SA), but not with either one alone, markedly induced pathogenesis-related genes $O s P R 1 b$ and probenazole-induced protein $1(P B Z 1)$. These effects were diminished by RNAi-knockdown of OsNPRI or WRKY45, the key regulators of the SA signaling pathway in rice, indicating that the effects of $\mathrm{CK}$ depend on these two regulators. Taken together, our data imply a coevolutionary rice-M. oryzae interaction, wherein $M$. oryzae probably elevates rice CK levels for its own benefits such as nutrient translocation. Rice plants, on the other hand, sense it as an infection signal and activate defense reactions through the synergistic action with SA.
\end{abstract}

Plant hormones play key signaling roles in the battle between host plants and their cognate microbial pathogens. Upon pathogen attack, plants modify and coordinate their hormone signaling pathways to activate defense responses so as to prevent pathogen infection (Grant and Jones 2009; Lopez et al. 2008; Robert-Seilaniantz et al. 2011). Pathogens, on the other hand, have evolved counteractive mechanisms that perturb the balance of hormone crosstalk by either producing plant hormones by themselves or altering the hormone biosynthesis pathways of host plants (Asselbergh et al. 2008; Grant and Jones 2009; Lopez et al. 2008; Robert-Seilaniantz et al. 2011). However, the

Corresponding author: C.-J. Jiang; Telephone and Fax: +81-29-838-8383; E-mail: cjjiang@affrc.go.jp

* The $\boldsymbol{e}$-Xtra logo stands for "electronic extra" and indicates that two supplementary figures and one supplementary table are published online.

(C) 2013 The American Phytopathological Society signaling network involving multiple hormones during plantpathogen interactions still remains rather elusive.

Cytokinins (CK) are a group of adenine-derived plant hormones that regulate diverse aspects of plant physiology. CK levels are spatiotemporally regulated by synthesis and catabolism. The rate-limiting step of CK synthesis is catalyzed by isopentenyl transferases (IPT), while CK catabolism is mainly catalyzed by cytokinin oxidase/dehydrogenases (CKX) that irreversibly degrade CK (Kudo et al. 2010; Sakakibara 2006). In Arabidopsis, CK are recognized by three hybrid histidine kinase receptors: specifically, ARABIDOPSIS HIS KINASE 2 (AHK2), AHK3, and AHK4/WOODENLEG 1 (WOL1)/CYTOKININ RESPONSE 1 (CRE1) (Argueso et al. 2010; Perilli et al. 2010). The CK signal is transmitted via a multistep phosphorelay pathway to the family of ARABIDOPSIS HIS PHOSPHOTRANSFER PROTEINS (AHP), which translocate into the nucleus and phosphorylate members of the type-B and type-A ARABIDOPSIS RESPONSE REGULATOR (ARR) protein family. Type-B ARR are transcription factors that activate primary CK-responsive genes, including type-A ARR. Type-A ARR negatively regulate CK signaling (Argueso et al. 2010; Perilli et al. 2010). In rice, certain genes have been recognized and characterized for IPT, $C K X$, histidine kinase (HK) from a $\mathrm{CK}$ receptor family, histidine-containing phosphotransmitter (HPt), and type-A and type-B RRs (Ashikari et al. 2005; Choi et al. 2012; Hirose et al. 2007; Ito and Kurata 2006; Jain et al. 2006; Sakamoto et al. 2006).

CK have been implicated in various plant-pathogen interactions, which are often manifested as disease symptoms associated with CK disorders (Choi et al. 2011; Grant and Jones 2009; Walters and McRoberts 2006). For example, infection of dicotyledonous plants by Agrobacterium tumefaciens causes crown gall tumors, which are attributed to the overproduction of CK and auxins by the IPT and iaaM/H genes, which are encoded on T-DNA integrated into the plant chromosome (Jameson 2000). The fungal pathogen Plasmodiophora brassicae, the causal agent of the Brassicaceae clubroot disease, has been shown to downregulate the CK degradation pathway during infection of Arabidopsis, and transgenic overexpression of CKX suppressed clubroot development, indicating the importance of CK in the pathogenicity of $P$. brassicae (Siemens et al. 2006). CK are also known to be associated with disease symptoms such as fasciations, senescence, and green islands formation (Choi et al. 2011; Grant and Jones 2009; Jameson 2000; Stes et al. 2011; Walters and McRoberts 2006). Thus, CK appear to act in favor of pathogen virulence in some pathosystems.

However, on the other hand, CK have also been demonstrated to play important roles in defense responses to patho- 
gen infections (Choi et al. 2011). It was recently shown that CK modulate the salicylic acid (SA) signaling pathway and promote resistance to the hemibiotrophic bacterial pathogens Pseudomonas syringae pv. tomato DC3000 and the biotrophic oomycete pathogen Hyaloperonospora arabidopsis isolate Noco2 in Arabidopsis (Argueso et al. 2012; Choi et al. 2010). The CK action was found to be mediated by the CK-activated transcription factor ARR2 that interacts with the SA response factor TGA3 and binds directly to the promoters of pathogenesis-related (PR) genes $P R 1$ and $P R 2$ to induce their transcription (Choi et al. 2010, 2011). Meanwhile, type-A ARR were found to negatively regulate SA-dependent basal immunity (Argueso et al. 2012). In tobacco (Nicotiana tabacum), transgenic plants with a bacterial ipt gene driven by a pathogeninducible promoter displayed enhanced resistance to virulent P. syringae pv. tabaci (Grosskinsky et al. 2011). The CK-mediated resistance is correlated with the upregulated synthesis of two major antimicrobial phytoalexins, scopoletin and capsidiol (Grosskinsky et al. 2011). Interestingly, the CK action in this system was independent of SA, a contrasting mechanism to that seen in Arabidopsis (Choi et al. 2010). It was also reported that elevated levels of endogenous CK led to accumulation of SA upon wounding and acidic PR-1 proteins and enhanced resistance to Tobacco mosaic virus in tobacco plants expressing rgpl, a rice gene for a small GTP-binding protein (Sano et al. 1994). Moreover, CK were also implicated in resistance to necrotrophic pathogens. Transgenic plants with increased CK levels exhibited delayed leaf senescence and attenuated disease symptoms of Botrytis cinerea infection in tomato (Swartzberg et al. 2008) and enhanced resistance to Alternaria brassicicola KACC40036 in Arabidopsis (Choi et al. 2010), respectively, whereas, in tobacco, increased CK levels had no effect on resistance to Sclerotinia sclerotiorum and even enhanced susceptibility to B. cinerea (Grosskinsky et al. 2011). Taken together, it seems that the role of CK varies in different pathosystems, reflecting the outcome of coevolutionary interactions between pathogens and their host plants.

SA plays a key role in the defense responses of both dicotyledonous and monocotyledonous plants (Kachroo and Kachroo 2007; Loake and Grant 2007). In rice, SA signaling is mediated by OsNPR1 and WRKY45 in a branched pathway (Shimono et al. 2007; Sugano et al. 2010). We have previously shown that abscisic acid (ABA) antagonizes SA signaling by suppressing the transcriptional induction of the OSNPRI and WRKY45 (Jiang et al. 2010). In this study, we report that high levels of CK accumulate in leaf blades following infection with the blast fungus Magnaporthe oryzae. In addition, we found that CK act synergistically with $\mathrm{SA}$ to activate $P R$ gene expressions in rice.

\section{RESULTS}

\section{CK accumulate in rice seedlings after blast infection.}

It has often been observed that pathogen infection leads to changes in hormone profiles in plants (Robert-Seilaniantz et al. 2011). To characterize possible changes in hormone levels during rice- $M$. oryzae interaction, we determined endogenous levels of indole-3-acetic acid (IAA), gibberellins (GA), CK, and $\mathrm{ABA}$ in leaf blades after inoculation with a compatible blast fungus (race 007.0). We took samples at three time points (36, 48, and $60 \mathrm{~h}$ postinoculation [hpi]), corresponding to the invasion stage of $M$. oryzae infection cycle, where the fungus differentiates bulbous infectious hyphae that colonize host tissues without visible damages (Ribot et al. 2008). The levels of three particular molecular species of CK-that is, $N^{6}-\left(\Delta^{2}\right.$-isopentenyl) adenine (iP), iP riboside (iPR), and iPR 5'-phosphates (iPRP) — were drastically increased in comparison with mock-treated controls (Table 1; Fig. 1). In particular, the iP levels in leaf blades were increased more than 100-fold 60 hpi (Fig. 1). After blast infection, the iP 7-glucoside (iP7G) and iP 9-glucoside (iP9G) levels also increased by approximately eight- and fourfold, respectively, indicating that part of the accumulated iP was converted to an inactive form, probably through a homeostatic mechanism. Limited changes were observed for the remaining CK species (Table 1). The IAA levels also increased approximately by threefold at $60 \mathrm{hpi}$ (Table 2). The levels of two nonbioactive GA (GA53 and GA44) slightly decreased, whereas ABA levels did not change significantly during the early time course of the experiment (Table 2).

In order to assess whether the increased CK level were a result of de novo production in rice seedlings, we examined the expression of the rice genes for IPT, an enzyme that catalyzes the first and rate-limiting step in CK biosynthesis, at

Table 1. Contents of cytokinins (CK) in leaf blades of rice seedlings after inoculation with blast fungus Magnaporthe oryzae ${ }^{\text {a }}$

\begin{tabular}{|c|c|c|c|c|c|c|}
\hline \multirow[b]{2}{*}{ CK } & \multicolumn{2}{|c|}{36 hpi } & \multicolumn{2}{|c|}{48 hpi } & \multicolumn{2}{|c|}{60 hpi } \\
\hline & Mock & Inoculation & Mock & Inoculation & Mock & Inoculation \\
\hline $\mathrm{tZ}$ & - & - & $3.11 \pm 0.27$ & $1.33 \pm 0.07 * *$ & $2.99 \pm 0.35$ & $2.63 \pm 0.60$ \\
\hline $\mathrm{tZR}$ & $0.13 \pm 0.02$ & $0.08 \pm 0.01$ & $0.32 \pm 0.03$ & $0.20 \pm 0.01$ & $0.36 \pm 0.02$ & $0.35 \pm 0.02$ \\
\hline tZRP & $0.46 \pm 0.09$ & $0.43 \pm 0.15$ & $1.19 \pm 0.07$ & $0.75 \pm 0.05$ & $1.23 \pm 0.21$ & $1.60 \pm 0.14$ \\
\hline $\mathrm{cZ}$ & $0.72 \pm 0.06$ & $0.92 \pm 0.18$ & $0.93 \pm 0.34$ & $1.58 \pm 0.45$ & $0.74 \pm 0.18$ & $0.90 \pm 0.29$ \\
\hline cZR & $0.61 \pm 0.01$ & $1.11 \pm 0.16$ & $0.42 \pm 0.10$ & $0.81 \pm 0.20$ & $0.56 \pm 0.07$ & $0.46 \pm 0.11$ \\
\hline cZRP & $0.38 \pm 0.02$ & $0.56 \pm 0.14 *$ & $0.32 \pm 0.07$ & $0.45 \pm 0.11$ & $0.30 \pm 0.10$ & $0.42 \pm 0.06$ \\
\hline iP & $0.21 \pm 0.02$ & $\mathbf{0 . 3 7} \pm \mathbf{0 . 0 8} *$ & $0.55 \pm 0.06$ & $5.06 \pm 0.89 * *$ & $0.80 \pm 0.10$ & $80.05 \pm 8.99 * *$ \\
\hline iPR & $0.1 \pm 0.01$ & $0.13 \pm 0.01$ & $0.32 \pm 0.02$ & $0.29 \pm 0.03$ & $0.44 \pm 0.13$ & $2.84 \pm 0.34 * *$ \\
\hline iPRP & $1.62 \pm 0.19$ & $3.18 \pm 0.45 * *$ & $7.66 \pm 0.54$ & $10.21 \pm 1.53$ & $6.36 \pm 1.36$ & $125.90 \pm 8.4^{* *}$ \\
\hline $\mathrm{tZ9G}$ & $3.37 \pm 0.3$ & $2.96 \pm 0.95$ & $3.9 \pm 0.62$ & $3.05 \pm 0.73$ & $3.63 \pm 0.42$ & $3.59 \pm 0.57$ \\
\hline tZOG & $0.47 \pm 0.03$ & $0.46 \pm 0.09$ & $0.78 \pm 0.09$ & $0.41 \pm 0.10^{*}$ & $0.78 \pm 0.08$ & $0.84 \pm 0.10$ \\
\hline cZOG & $245.01 \pm 32$ & $260.00 \pm 21$ & $262.00 \pm 13$ & $270.88 \pm 41$ & $227.85 \pm 20$ & $288.87 \pm 23$ \\
\hline cZROG & $5.40 \pm 0.48$ & $7.16 \pm 0.98$ & $5.97 \pm 0.41$ & $7.00 \pm 0.50$ & $5.58 \pm 0.64$ & $7.23 \pm 0.78$ \\
\hline cZRPsOG & $0.98 \pm 0.10$ & $1.52 \pm 0.15$ & $1.00 \pm 0.11$ & $1.25 \pm 0.17$ & $0.73 \pm 0.04$ & $0.95 \pm 0.14$ \\
\hline iP7G & $0.02 \pm 0.00$ & - & - & $0.03 \pm 0.00$ & $0.02 \pm 0.00$ & $0.17 \pm 0.08$ \\
\hline iP9G & $0.63 \pm 0.15$ & $0.65 \pm 0.18$ & $0.69 \pm 0.36$ & $0.89 \pm 0.25$ & $0.55 \pm 0.10$ & $2.26 \pm 0.19$ \\
\hline
\end{tabular}

${ }^{\text {a }}$ Samples were taken at 36,48 , and $60 \mathrm{~h}$ postinoculation (hpi). Values are represented as mean \pm standard deviation in picomoles per gram of fresh weight $(n=4$ biological replicates). Abbreviations: tZ, trans zeatin; tZR, tZ riboside; tZRP, tZR phosphates; cZ, cis zeatin; cZR, cZ riboside; cZRP, cZR phosphates; iP, $N^{6}$-( $\Delta^{2}$-isopentenyl) adenine; iPR, iP riboside; iPRP, iPR phosphates; tZ9G, tZ 9-glucoside; tZOG, tZ $O$-glucoside; cZOG, cZ $O$-glucoside; cZROG, cZR O-glucoside; cZRPsOG, cZPs O-glucoside; iP7G, iP 7-glucoside; and iP9G, iP 9-glucoside. Dihydrozeatin (DZ), DZ riboside (DZR), DZR phosphates (DZRP), tZ 7-glucoside (tZ7G), DZ 9-glucoside (DZ9G), tZR $O$-glucoside (tZROG), and tZRPs $O$-glucoside (tZRRsOG) were not detected. Asterisks indicate significant difference from the mock-treated plants ( $t$ test, $*$ and $* *$ indicate $P<0.05$ and 0.01 , respectively). CK molecule species that are significantly increased after blast inoculation appear in bold. 
various time points between 6 and 72 hpi. There are eight $I P T$ genes in the rice genome (OSIPT1 to -8), of which OSIPT6 is a pseudogene (Sakamoto et al. 2006). The quantitative reversetranscriptase polymerase chain reaction (qRT-PCR) analysis of

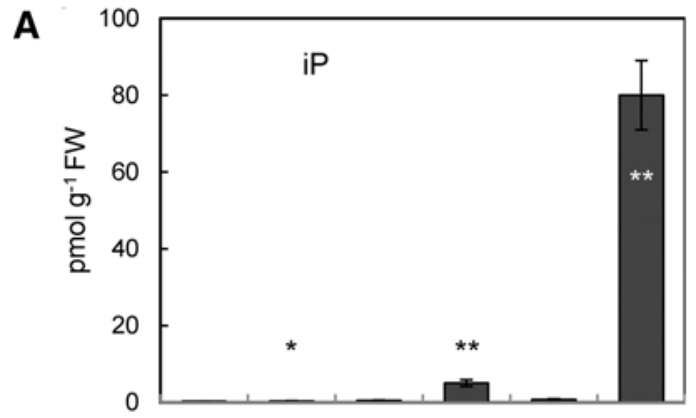

B

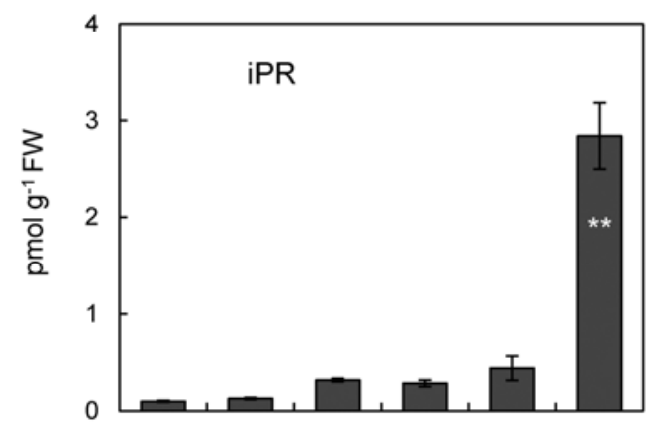

C

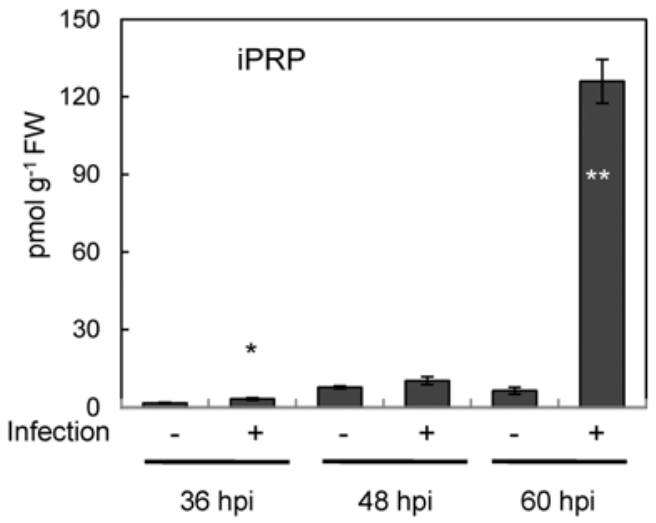

Fig. 1. Accumulation of cytokinins (CK) in Magnaporthe oryzae-infected rice. Three CK species-namely, $N^{6}-\left(\Delta^{2}\right.$-isopentenyl) adenine (iP), iP riboside (iPR), and iPR $5^{\prime}$-phosphates (iPRP)—were massively accumulated in rice leaf blades after inoculation with blast fungus $M$. oryzae. Increased accumulation of CK was seen at $36 \mathrm{~h}$ postinoculation (hpi) and continued thereafter. Values are represented as mean \pm standard deviation $(n=4$ biological replicates) in picomoles per gram of fresh weight. Asterisks indicate significant difference from the mock-treated plants $(t$ test, $*$ and $* *$ indicate $P<$ 0.05 and 0.01 , respectively). these genes (except for OsIPT6) showed that none of them was upregulated before the $\mathrm{CK}$ levels increased (Supplementary Fig. S1). We also examined the expression levels of the rice genes for $C K X$. There are 11 putative $C K X$ genes (OsCKX1 to OsCKX11) present in the rice genome (Ashikari et al. 2005), and none of them exhibited expression changes before the $\mathrm{CK}$ levels increased (Supplementary Fig. S2). These data suggest that accumulation of $\mathrm{CK}$ following blast infection does not depend on the transcriptional regulation of OsIPT or OsCKX.

\section{CK signaling is activated by blast infection.}

To monitor the activation of CK signaling during blast infection, transgenic rice plants expressing the $\beta$-glucuronidase (GUS) reporter gene under the control of a CK-responsive OsRR6 promoter (OSRR6::GUS) were inoculated with the blast fungus, and leaf blades were subjected to histochemical staining for GUS activity $48 \mathrm{hpi}$. The result showed that CK signaling was activated around blast lesions of infection sites as shown by GUS staining (Fig. 2B), consistent with the increased CK levels induced by blast infection (Table 1; Fig. 1).
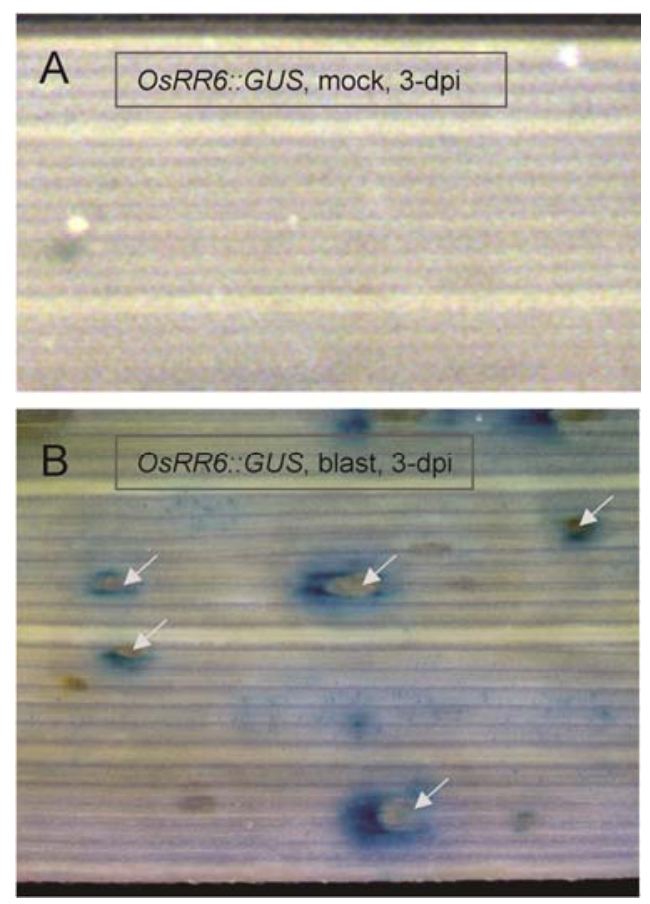

Fig. 2. Cytokinin (CK) signaling was activated by blast infection. OsRR6: $\beta$-glucuronidase (GUS) rice seedlings were $\mathbf{A}$, treated with mock or $\mathbf{B}$, inoculated with blast fungus and the leaf blades were subjected to histochemical staining for GUS activity at 3 days postinoculation (dpi). CK signaling was activated around the blast lesions of infection sites as shown by GUS staining (B). Arrows indicate blast lesions.

Table 2. Hormone contents in leaf blades of rice seedlings after inoculation with blast fungus Magnaporthe oryza $e^{\mathrm{a}}$

\begin{tabular}{|c|c|c|c|c|c|c|}
\hline \multirow[b]{2}{*}{ Hormones $^{b}$} & \multicolumn{2}{|c|}{36 hpi } & \multicolumn{2}{|c|}{48 hpi } & \multicolumn{2}{|c|}{60 hpi } \\
\hline & Mock & Inoculation & Mock & Inoculation & Mock & Inoculation \\
\hline IAA & $63.8 \pm 4.1$ & $83.0 \pm 13.5$ & $43.8 \pm 4.6$ & $60.5 \pm 2.8^{*}$ & $28.5 \pm 2.4$ & $88.8 \pm 17.4 * *$ \\
\hline GA1 & u.q. & u.q. & u.q. & u.q. & u.q. & u.q. \\
\hline GA19 & $78.3 \pm 16.2$ & $89.9 \pm 15.1$ & $49.9 \pm 7.9$ & $92.6 \pm 21.8$ & $55.7 \pm 8.9$ & $50.9 \pm 12.3$ \\
\hline GA24 & $5.2 \pm 1.9$ & $4.3 \pm 1.1$ & $3.8 \pm 1.8$ & $3.0 \pm 1.3$ & $3.8 \pm 1.7$ & $5.8 \pm 1.5$ \\
\hline GA44 & $3.3 \pm 0.2$ & $3.8 \pm 0.99$ & $5.6 \pm 0.7$ & 2.6. $\pm 0.4^{* *}$ & $4.7 \pm 0.5$ & $2.2 \pm 0.4^{* *}$ \\
\hline GA53 & $42.0 \pm 8.3$ & $40.3 \pm 8.7$ & $18.5 \pm 3.6$ & $16.6 \pm 4.3$ & $31.6 \pm 6.3$ & $13.3 \pm 1.1 * *$ \\
\hline $\mathrm{ABA}$ & $484.8 \pm 144.7$ & $401.3 \pm 65.3$ & $278.0 \pm 93.8$ & $218.8 \pm 54.3$ & $237.1 \pm 62.4$ & $287.5 \pm 92.9$ \\
\hline
\end{tabular}

${ }^{a}$ Samples were taken at 36,48 , and $60 \mathrm{~h}$ postinoculation (hpi); u.q. = under quantification limit. Values are represented as mean \pm standard deviation in picomoles per gram of fresh weight. ( $n=4$ biological replicates). Asterisks indicate significant difference from the mock-treated plants $(t$ test, $*$ and $* *$ indicate $P<$ and 0.01 , respectively).

${ }^{\mathrm{b}}$ IAA $=$ indole-3-acetic acid, $\mathrm{GA}=$ gibberellin, and $\mathrm{ABA}=$ abscisic acid . 


\section{M. oryzae produces plant hormones.}

Many bacterial and fungal pathogens are capable of producing plant hormones by themselves (Robert-Seilaniantz et al. 2007). The sharp increase in CK levels after blast infection prompted us to determine the plant hormones in hyphae, conidia, and culture filtrates of $M$. oryzae. We chose two blast races, 107.0 and 102.0, for the hormone determination. Substantial amounts of various CK, including iP, iPR, and iPRP, accumulated in both hyphae (mycelium) and conidia (Table 3 ). The CK were also detected in culture filtrates (Table 3), indicating that the hormones produced in fungus hyphae were released into the culture medium. ABA (Jiang et al. 2010) and IAA (Table 4) were also detected in all the samples. On the other hand, no substantial amount of any GA species was detected.

\section{CK act synergistically with SA to activate defense gene expression.}

To obtain an insight about the physiological significance of $\mathrm{CK}$ accumulation during blast infection, we analyzed the effects of CK on the SA-mediated signaling pathway by determining expression of PR genes $O s P R I b$ and probenazole-induced protein $1(P B Z 1)$. In rice-leaf segments, OsPRIb expression was induced after $24 \mathrm{~h}$ of treatment with SA (Agrawal et al. 2000). $P B Z 1$ is a rice $P R 10$ that was identified after $72 \mathrm{~h}$ of treatment with probenazole (PBZ, an SA analog) (Midoh and Iwata 1996). Treating leaf blades with CK or SA for $8 \mathrm{~h}$ had no significant effect on these $P R$ gene expressions (Fig. 3A and B). However, treatment of the leaf blades with both $\mathrm{CK}$ and SA markedly induced $O S P R I b$ and $P B Z 1$ levels (Fig. 3C and D). These results show that the combined treatment with $\mathrm{CK}$ and SA accelerates the induction of these two $P R$ genes, indicating the synergistic action of $\mathrm{CK}$ and $\mathrm{SA}$ on $P R$ gene induction. Such effects were diminished by RNAi-knockdown of OSNPRl or WRKY45 (Fig. 4A and B), the key regulators of the SA signaling pathway in rice. Conversely, in transgenic rice plants overexpressing $W R K Y 45, \mathrm{CK}$ treatment alone induced the expression of $O s P R 1 b$ and $P B Z 1$ (Fig. 4C and D). In addition, the CK did not further increase SA-induced expression levels of OsNPRl and WRKY45 (Jiang et al. 2010). These data indicate that the synergistic actions of CK and SA depend on OsNPR1 and WRKY45, and that the CK act on or downstream of these two regulators. None of the other hormones tested showed effects similar to those of CK (Fig. 3).

To further investigate the effects of endogenous CK levels on blast resistance, we generated transgenic rice lines by using the rice $C K X 2$ gene (Ashikari et al. 2005) under the control of a GVG system ( $G V G:: C K X 2)$, in which $C K X 2$ expression is induced by treatment with dexamethasone (Aoyama and Chua 1997). GVG::CKX2 plants exhibited a leaky expression of $C K X 2$ (Fig. 5A) and, hence, very poor rooting and plant growth. In some of these transgenic lines, we observed the growth of adventitious roots from the surface of leaf blades (Fig. 5Ba) and leaf sheaths (Fig. 5Bb), indicating a low ratio of $\mathrm{CK}$ to auxins in these transgenic lines. We selected two transgenic lines (numbers 3 and 9) that had relatively low $C K X 2$ expression levels and moderate phenotypes for the subsequent experiments (Fig. 5A). In these lines, the CK inducibility of OsRR6 was decreased significantly (Fig. 5C), indicating the suppression of $\mathrm{CK}$ signaling in these plants. Compared with nontransgenic controls, $G V G \because: C K X 2$ plants with blast infection revealed no significant difference in disease incidence (Fig. 5D). The blast-induced levels of $O s P R 1 b$ were lower in $G V G: \because C K X 2$ plants than in the nontransgenic controls (Fig. 5E).

It was shown in Arabidopsis that CK treatment altered susceptibility to $H$. arabidopsis Noco 2 in a dose-dependent manner (Argueso et al. 2012). Low concentration $(\leq 10 \mu \mathrm{M})$ of bezyl adenine (BA) enhanced susceptibility, while high concentration of BA $(100 \mu \mathrm{M})$ primed SA-dependent defense responses (Argueso et al. 2012). To examine this phenomenon in rice, we spray treated wild-type rice seedlings with different concentrations of kinetin or iP, followed by blast inoculation

Table 3. Contents of cytokinins (CK) in hyphae and conidia of Magnaporthe grisea and culture medium ${ }^{\mathrm{a}}$

\begin{tabular}{|c|c|c|c|c|c|c|}
\hline \multirow[b]{2}{*}{ CK } & \multicolumn{3}{|c|}{ Blast race 007.0 } & \multicolumn{3}{|c|}{ Blast race 102.0 } \\
\hline & Hyphae & Filtrate & Conidia & Hyphae & Filtrate & Conidia \\
\hline $\mathrm{tZ}$ & - & - & $0.23 \pm 0.07$ & - & - & $0.23 \pm \mathrm{nc}$ \\
\hline $\mathrm{tZR}$ & - & - & $0.35 \pm 0.06$ & - & - & $0.12 \pm \mathrm{nc}$ \\
\hline tZRP & $0.12 \pm 0.03$ & - & $0.25 \pm 0.08$ & - & - & $0.17 \pm \mathrm{nc}$ \\
\hline cZ & $1.14 \pm 0.16$ & - & $4.04 \pm 0.20$ & $0.46 \pm 0.14$ & - & $5.29 \pm \mathrm{nc}$ \\
\hline cZR & $3.09 \pm 0.21$ & $0.73 \pm 0.05$ & $8.47 \pm 0.15$ & $1.93 \pm 1.22$ & $0.51 \pm 0.04$ & $5.69 \pm \mathrm{nc}$ \\
\hline cZRP & $4.94 \pm 0.48$ & $0.14 \pm 0.07$ & $4.17 \pm 0.49$ & $3.86 \pm 2.63$ & $0.61 \pm 0.03$ & $6.22 \pm \mathrm{nc}$ \\
\hline iP & $0.47 \pm 0.07$ & $0.54 \pm 0.06$ & $2.25 \pm 1.08$ & $3.89 \pm 0.35$ & $0.64 \pm 0.02$ & $1.42 \pm \mathrm{nc}$ \\
\hline iPR & $0.83 \pm 7$ & $0.78 \pm 0.10$ & $1.72 \pm 0.22$ & $18.80 \pm 2.37$ & $1.62 \pm 0.11$ & $1.08 \pm \mathrm{nc}$ \\
\hline iPRP & $5.82 \pm 0.49$ & $4.65 \pm 0.57$ & $2.29 \pm 0.05$ & $10.48 \pm 2.27$ & $5.26 \pm 0.20$ & $2.81 \pm \mathrm{nc}$ \\
\hline $\mathrm{tZ7G}$ & - & $0.11 \pm 0.02$ & $0.59 \pm 0.44$ & $0.24 \pm 0.07$ & - & - \\
\hline cZOG & - & $9.30 \pm 1.02$ & $2.71 \pm 0.34$ & $1.89 \pm 0.65$ & $13.17 \pm 2.83$ & $0.41 \pm \mathrm{nc}$ \\
\hline tZROG & - & - & $0.16 \pm \mathrm{nc}$ & - & - & - \\
\hline cZROG & $0.21 \pm 0.02$ & $0.17 \pm 0.10$ & $2.58 \pm 0.24$ & $0.08 \pm 0.03$ & $0.08 \pm 0.01$ & $0.52 \pm \mathrm{nc}$ \\
\hline cZRPsOG & $0.05 \pm \mathrm{nc}$ & $0.03 \pm 0.02$ & $0.14 \pm 0.04$ & - & - & $0.07 \pm \mathrm{nc}$ \\
\hline iP7G & $30.88 \pm 2.89$ & $21.95 \pm 1.94$ & $0.89 \pm 0.46$ & $5.95 \pm 0.58$ & $3.54 \pm 0.04$ & $0.45 \pm \mathrm{nc}$ \\
\hline
\end{tabular}

${ }^{a}$ Values are represented as mean \pm standard deviation (SD) in picomoles per gram of fresh weight $(n=4$ biological replicates); nc $=$ the SD was not calculated because there were only two samples. Abbreviations: tZ, trans zeatin; tZR, tZ riboside; tZRP, tZR phosphates; cZ, cis zeatin; cZR, cZ riboside; cZRP, cZR phosphates; iP, $N^{6}$-( $\Delta^{2}$-isopentenyl) adenine; iPR, iP riboside; iPRP, iPR phosphates; tZ7G, tZ 7-glucoside; cZOG, cZ $O$-glucoside; cZROG, cZR $O$-glucoside; cZRPsOG, cZPs $O$-glucoside; and iP7G, iP 7-glucoside. Dihydrozeatin (DZ), DZ riboside (DZR), DZR phosphates (DZRP), tZ 9-glucoside (tZ9G), tZR $O$-glucoside (tZROG), tZRPs $O$-glucoside (tZRRsOG), DZ 9-glucoside (DZ9G), and iP 9-glucoside (iP9G) were not detected.

Table 4. Indole-3-acetic acid contents in hyphae and conidia of Magnaporthe grisea and culture medium ${ }^{\mathrm{a}}$

\begin{tabular}{lccc}
\hline Blast races & Hyphae & Filtrate & Conidia \\
\hline 007.0 & $620.77 \pm 87.18$ & $361.25 \pm 64.44$ & $351.57 \pm 10.24$ \\
102.0 & $351.16 \pm 35.94$ & $290.48 \pm 11.93$ & $97.66 \pm \mathrm{nc}$ \\
\hline
\end{tabular}

${ }^{a}$ Values are represented as mean \pm standard deviation (SD) in picomoles per gram of fresh weight. ( $n=4$ biological replicates); nc indicates that the SD was not calculated because there were only two samples. 
after $24 \mathrm{~h}$. No significant differences in blast resistance were observed across the treatments (Fig.5F), although it was likely that the resistance was slightly compromised in most treatments.

\section{DISCUSSION}

CK play crucial roles in diverse processes of plant growth and development (Kudo et al. 2010; Perilli et al. 2010; Sakamoto et al. 2006) as well as in biotic (Choi et al. 2011) and abiotic (Ha et al. 2012; Peleg et al. 2011) stress responses. It has been shown that increased CK levels enhance disease resistance to $P$. syringae pv. tomato DC3000 (Choi et al. 2010)

\section{A}

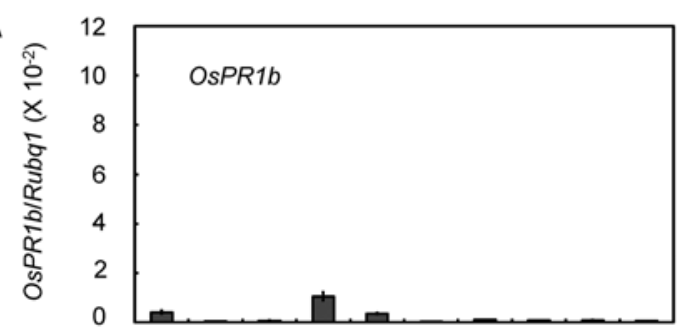

B

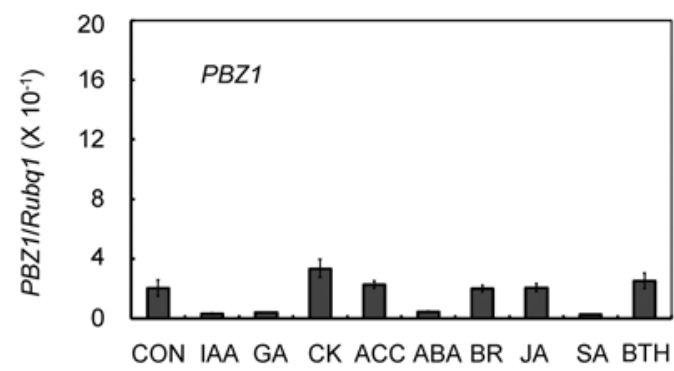

C

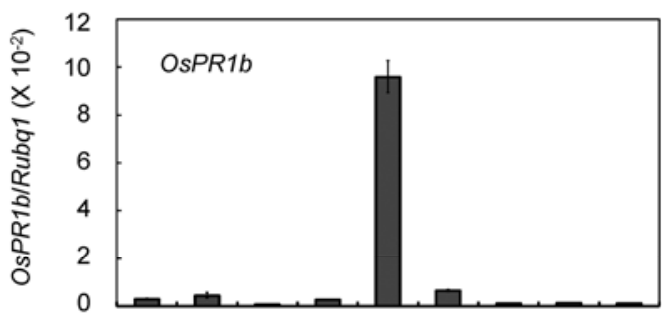

D

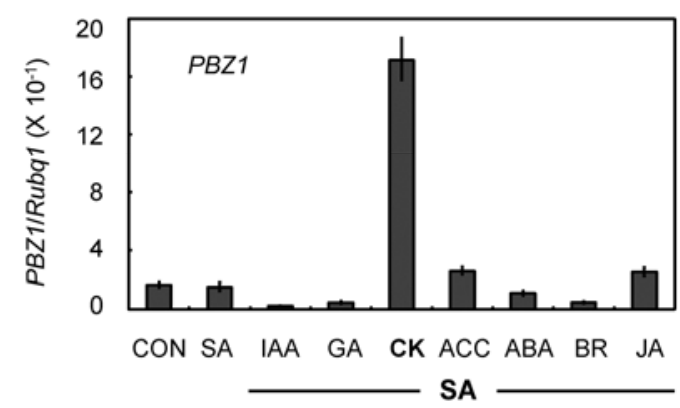

Fig. 3. Cytokinins (CK) act synergistically with salicylic acid (SA) to activate defense gene expression. Leaf segments from rice seedlings were treated in solutions containing various plant hormones under light at $30^{\circ} \mathrm{C}$ for $8 \mathrm{~h}$. Transcript levels of $O S P R I b$ and $P B Z 1$ were determined by quantitative reverse-transcriptase polymerase chain reaction analysis using rice ubiquitin 1 (Rubq1) as an internal control. Mean values from three determinations with standard deviation are shown. $\mathbf{A}$ and $\mathbf{B}$, Single hormone treatments; $\mathbf{C}$ and $\mathbf{D}$, co-treatments of SA with various hormones. SA, $1 \mathrm{mM}$; BTH, benzothiadiazole $S$-methyl ester $(50 \mu \mathrm{M})$; IAA, indole-3-acetic acid $(50 \mu \mathrm{M})$; GA, gibberellin $(50 \mu \mathrm{M})$; $\mathrm{CK}$, kinetin $(50 \mu \mathrm{M})$; ACC, 1 aminocyclopropane-1-carboxylic acid $(50 \mu \mathrm{M}) ; \mathrm{ABA}$, abscisic acid $(50$ $\mu \mathrm{M})$; BR, brassinolide $(2 \mu \mathrm{M}) ; \mathrm{JA}$, jasmonic acid $(50 \mu \mathrm{M})$; CON, mock treatment. and H. arabidopsis Noco2 (Argueso et al. 2012) in Arabidopsis, and to $P$. syringae pv. tabaci (Grosskinsky et al. 2011) and Tobacco mosaic virus (Sano et al. 1994) in tobacco. In rice, CK promoted production of diterpenoid phytoalexins (Ko et al. 2010) and acted synergistically with the SA pathway to activate defense gene expression (Fig. 3). On the other hand, transgenic overexpression of $C K X$ suppressed the development of clubroot disease in Arabidopsis, where CK are associated with the pathogenicity of $P$. brassicae (Siemens et al. 2006). Moreover, it has also been shown that increased CK levels enhance grain yield (Ashikari et al. 2005) and drought tolerance (Peleg et al. 2011) in rice plants. These findings imply that CK

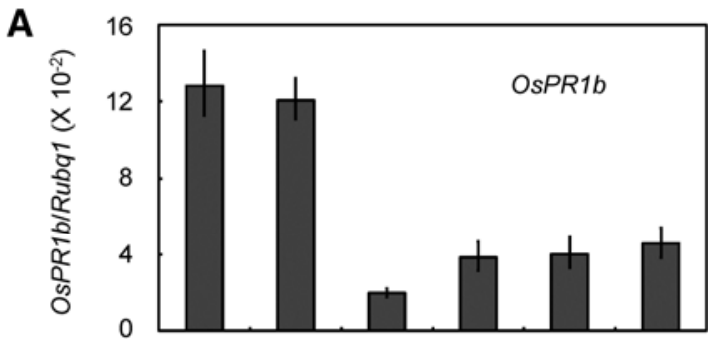

B

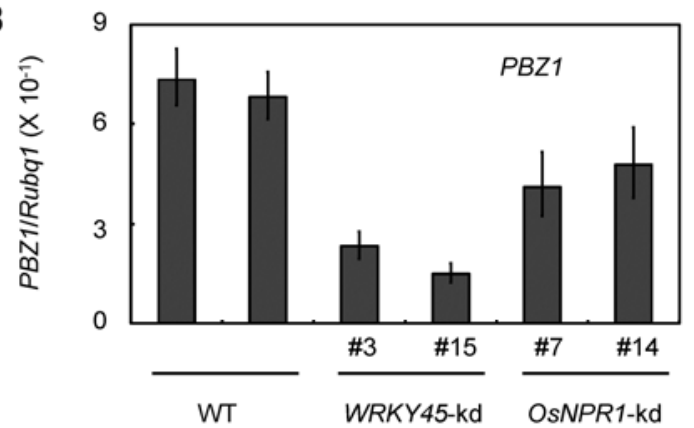

C

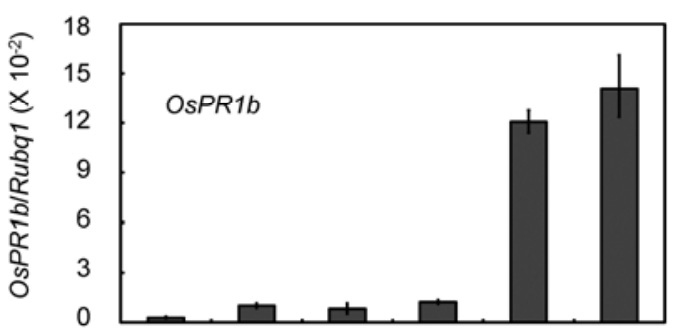

D

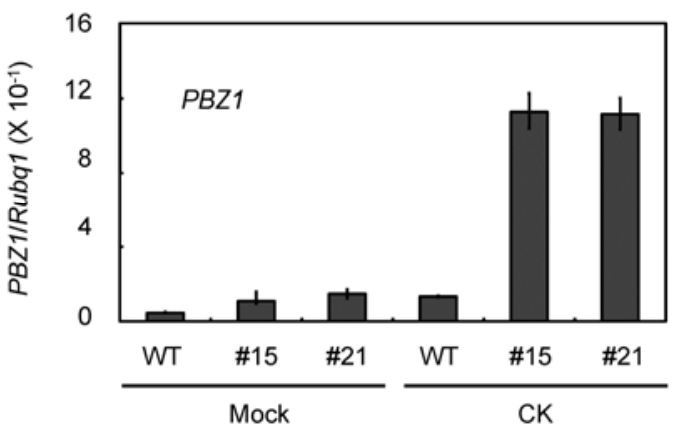

Fig. 4. Inductions of $O S P R 1 b$ and probenazole-induced protein 1 (PBZ1) by cytokinin (CK) and salicylic acid (SA) co-treatment depend on OSNPRI and WRKY45. A and B, Leaf segments from wild-type (WT), WRKY45-kd (number 3 and number 15), OsNPRI-kd (number 7 and number 14), and WRKY45-ox (number 15 and number 21) rice seedlings were treated with both CK and SA or $\mathbf{C}$ and $\mathbf{D}, \mathrm{CK}$ alone under light at $30^{\circ} \mathrm{C}$ for $8 \mathrm{~h}$, and the transcript levels of $O S P R 1 b$ and $P B Z 1$ were determined by quantitative reverse-transcriptase polymerase chain reaction analysis using rice ubiquitin 1 (Rubq1) as an internal control. Mean values from three determinations with standard deviation are shown. CK: $50 \mu \mathrm{M}$; SA: $1 \mathrm{mM}$. 
metabolism and signaling pathways may serve as an important engineering target for agricultural application.

Many fungal and bacterial phytopathogens have been known to disturb hormone profiles either by producing hormones by themselves or by modulating hormone synthesis pathways in host plants (Grant and Jones 2009; Robert-Seilaniantz et al. 2007). In this study, we revealed a massive and selective accumulation of three CK species (i.e., iP, iPR, and iPRP) in leaf blades of rice seedlings after infection with the blast fungus $M$. oryzae (Table 1; Fig. 1). In particular, the iP was increased up to100-fold higher than the mock control. The iP is considered to be the major bioactive CK in rice (Sakamoto et al. 2006).
Consistent with the CK accumulation, CK signaling was activated in response to blast infection, as shown by the GUS activity observed around blast lesions at the infection sites in OsRR6::GUS seedlings (Fig. 2). M. oryzae is a hemibiotrophic fungus that has a biotrophic phase during initial cell invasion (Kankanala et al. 2007; Ribot et al. 2008; Talbot et al. 1993). The infection cycles of hemibiotrophic fungi have been well studied in Colletotrichum spp. and revealed that Colletotrichum spp. have the biotrophic phase confined to the first infected host cells and then switch to necrotrophic phase, creating rapidly expanding necrotic lesions (Perfect et al. 1999; Ribot et al. 2008). In comparison, M. oryzae uses an extended
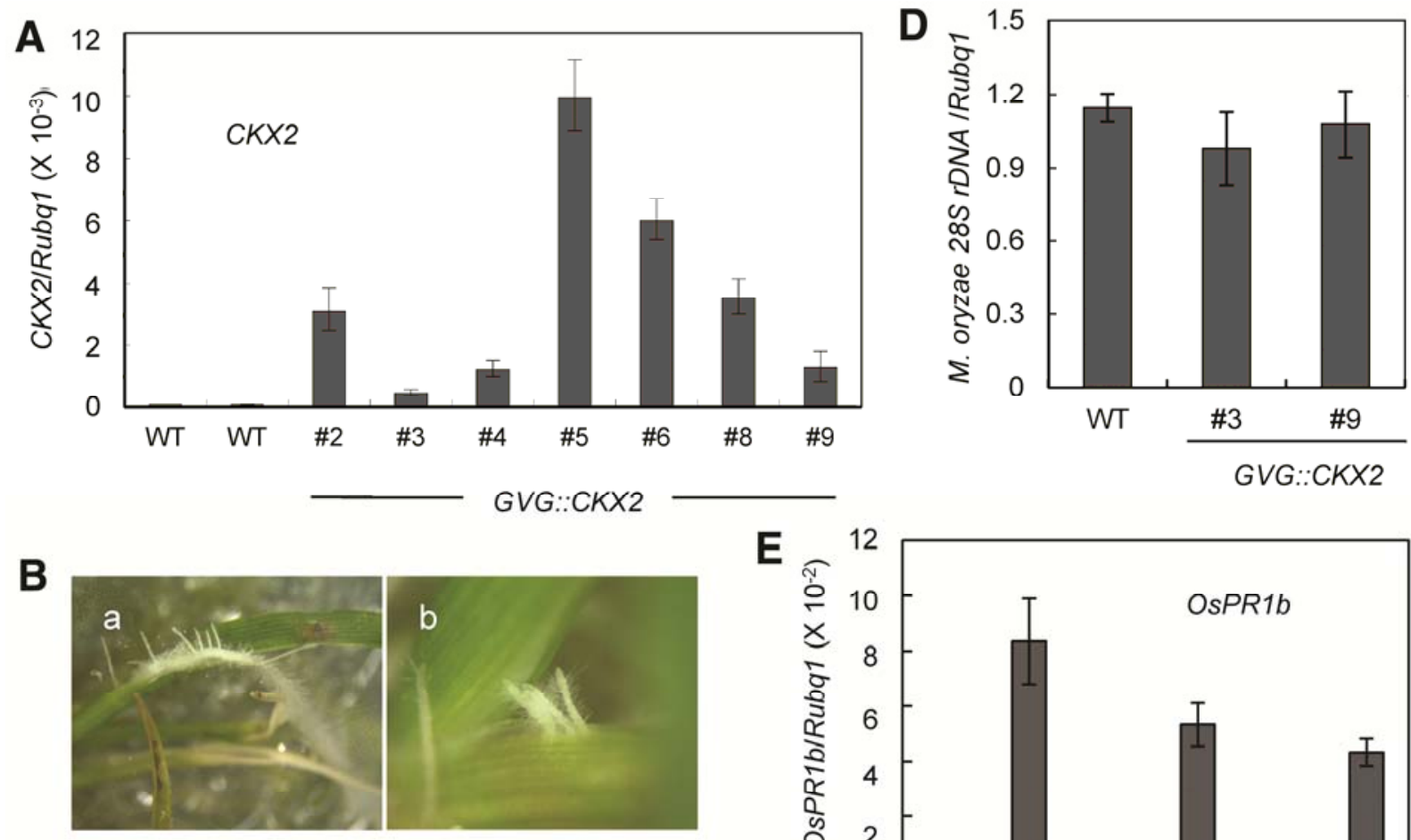

E
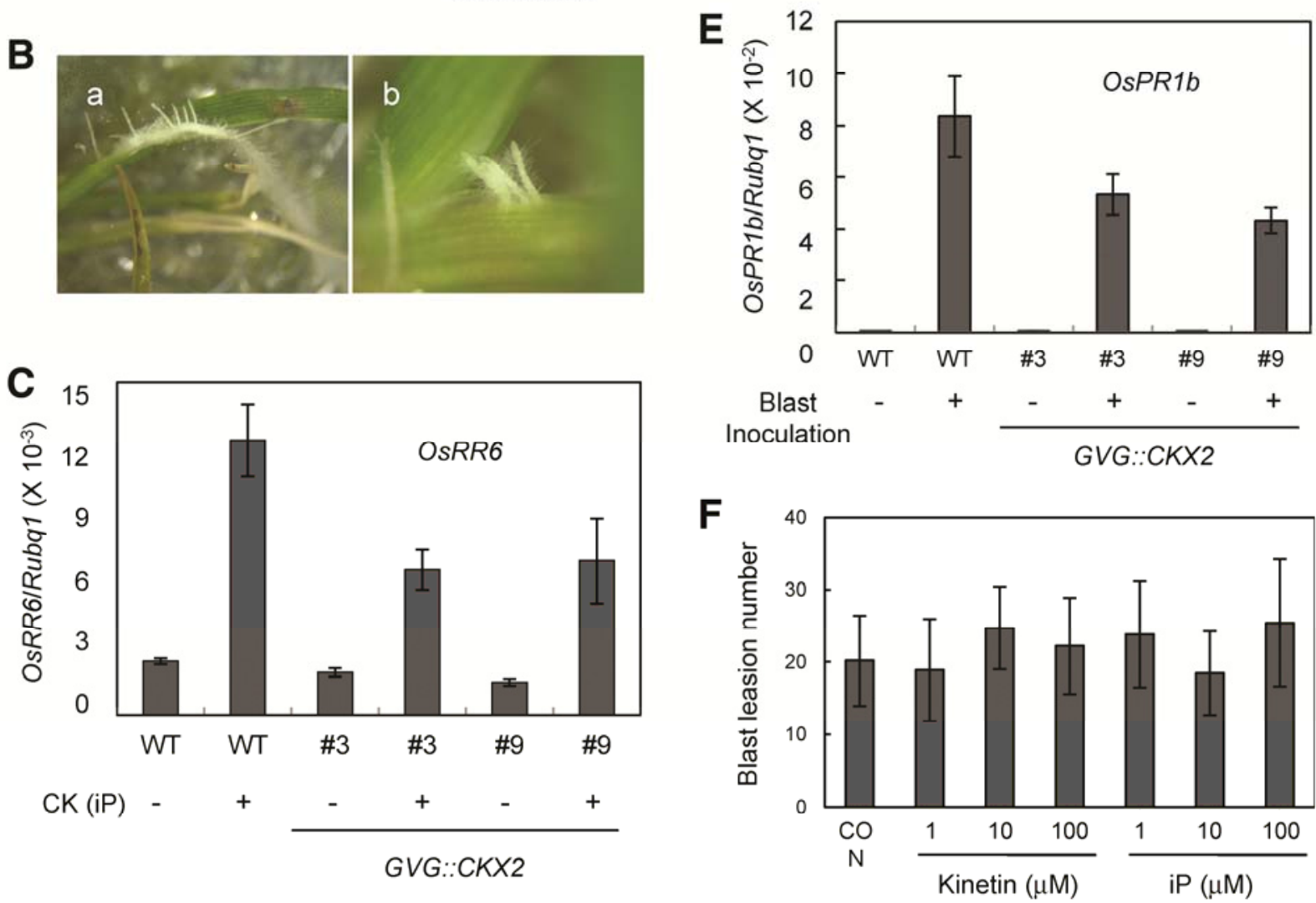

Fig. 5. Blast resistance was not altered in $G V G:: C K X 2$ transgenic or cytokinin (CK)-treated wild-type (WT) rice plants. A, Transcript levels of $C K X 2$ in GVG::CKX2 transgenic rice lines were determined by quantitative reverse-transcriptase polymerase chain reaction analysis using rice ubiquitin 1 (Rubq1) as an internal control. B, Adventitious roots were observed growing out form the surface of leaf blade (a) and leaf sheath (b). C, Segments from WT and $G V G:: C K X 2$ (number 3 and number 9) rice seedlings were treated with mock (-) or CK (+) $\left(N^{6}\right.$ - $\left(\Delta^{2}\right.$-isopentenyl) adenine (iP), 50 $\left.\mu \mathrm{M}\right)$ under light at $30^{\circ} \mathrm{C}$ for $8 \mathrm{~h}$, and the transcript levels of $O s R R 6$ were determined. D, WT and $G V G:: C K X 2$ (number 3 and number 9) rice seedlings were inoculated with Magnaporthe oryzae, and the levels of $M$. oryzae $28 \mathrm{~S}$ rDNA at 6 days postinoculation (dpi) and E, OsPR1b transcript at 3 dpi were determined. Mean values from three determinations with standard deviation are shown. F, WT rice seedlings were spray treated with indicated concentration of dimethyl sulfoxide (Con), kinetin, or iP $24 \mathrm{~h}$ prior to blast inoculation. Mean values for lesion number per 10-cm middle region of leaf blade with standard deviation are shown. 
biotrophic invasion strategy, with the fungal growth front always being ahead of visible lesions, even during the late stages (96 hpi) of infection (Berruyer et al. 2006; Kankanala et al. 2007). Thus, our observation may indicate that CK accumulation and CK signaling activation occur in the growth front of $M$. oryzae. Furthermore, the expression of IPT and $C K X$ genes did not change after blast infection. In contrast, a variety of CK species (including iP, iPR, and iPRP) were detected in the hyphae, conidia, and culture filtrates (Table 3 ). This indicates that $M$. oryzae is able to produce its own CK. On the basis of these data, it might be speculated that $\mathrm{CK}$ accumulations found in leaf blades after blast infection originate from $M$. oryzae. However, the $\mathrm{CK}$ levels detected in $M$. oryzae fungus were only approximately a few percent of that in blast-infected leaves, which may fail to fully account for the massive CK accumulations. Therefore, alternatively, it is possible that CK accumulate because of the post-transcriptional activation of rice IPT proteins in response to blast infection, as has been recorded for IPT3 in Arabidopsis, which undergoes post-translational activation by farnesylation (Galichet et al. 2008).

In addition to CK, ABA (Jiang et al. 2010) and IAA were also detected in $M$. oryzae (Table 4). The IAA levels were increased by several fold in leaf blades after blast infection (Table 2 ). It has been shown that auxins negatively regulate defense responses to Xanthomonas oryzae pv. oryzae and M. oryzae through activation of expansin-mediated cell wall loosening (Ding et al. 2008; Domingo et al. 2009). Our findings imply that $M$. oryzae fungus may stimulate auxin accumulation in the host leaves to facilitate its infection in the initial interaction stages. On the other hand, the ABA levels in leaf blades were not changed during the early stages after blast infection (Table 2 ). This was contrary to our expectation, because we previously found that ABA interacts antagonistically with SA in defense induction (Jiang et al. 2010) and the reduction of ABA levels by transgenic expression of OsABA8oxl gene encoding an ABA 8'-hydroxylase enhanced blast resistance (Yazawa et al. 2012) in rice. It may be possible that, even though ABA levels increase around the infection sites, the induced levels were too low to be detectable in this study.

Hormone crosstalk is known to regulate the outcome of plant-pathogen interactions (Grant and Jones 2009; Lopez et al. 2008; Robert-Seilaniantz et al. 2011). Many phytopathogens are capable of interfering with the hormone balances in host plants for their own benefit (Grant and Jones 2009). Infection of biotrophic or hemibiotrophic fungal pathogens often causes delayed senescence of infected sites to form "green islands" that have strong sink activity to accumulate various nutrients and, therefore, support pathogen growth (Ashby 2000; RobertSeilaniantz et al. 2011; Walters and McRoberts 2006). Significantly higher levels of biologically active CK cis-zeatin9-riboside and cis-zeatin-9-riboside-5'-monophosphate were detected in Colletotrichum graminicola-caused green islands compared with surrounding senescencing tissues in maize leaves (Behr et al. 2010, 2012). It is generally known that CK enhance sink activity by activating invertase and hexose transporter activity (Robert-Seilaniantz et al. 2011; Walters and McRoberts 2006). In this study, we found that CK accumulate in response to blast infection and that $\mathrm{CK}$ signaling is activated primarily around blast infection sites (Table 1; Fig. 2). Previous reports have shown that pretreatment with a high dose of kinetin partially decreases rice blast resistance (Matsumoto 1980). These data imply that CK accumulation benefits fungal infection or growth by, for example, mobilizing nutrients toward the pathogen-residing sites in rice. However, on the other hand, we also revealed that $\mathrm{CK}$ interact synergistically with SA to activate $P R$ gene expressions (Fig. 3C and D). This result indicates that $\mathrm{CK}$ are positively involved in rice defense reactions in rice. Similar action of CK was reported recently in Arabidopsis, where CK modulated the SA signaling pathway and promoted resistance to $P$. syringae pv. tomato DC3000 and H. arabidopsis Noco2 (Argueso et al. 2012; Choi et al. 2010). Moreover, it was shown that CK treatment induces production of the major diterpenoid phytoalexins-momilactones and phytocassanes - in rice (Ko et al. 2010). The levels of these phytoalexins were shown to increase significantly in leaf blades of rice in response to blast infection, and treatment with momilatone A suppressed blast fungal growth in planta and in vitro, demonstrating an important role for phytoalexins in blast resistance (Hasegawa et al. 2010). Taken together, these data suggest that CK may play two opposing roles in rice- $M$. oryzae interactions. On the one hand, $M$. oryzae elevates rice $\mathrm{CK}$ levels during the initial stages of infection for its own benefits such as nutrient translocation. Rice plants, on the other hand, sense the CK accumulation as an infection signal and activate defense responses by acting synergistically with SA. In support of this notion, neither transgenic expression of rice $C K X 2$ nor CK treatment of wild-type rice seedlings significantly altered blast resistance (Fig. 5D and F). This may be due to the fact that the initial effect of lowered or enhanced CK signaling pathway is partially neutralized by its opposing actions in the later stages.

The mode of $\mathrm{CK}$ function in rice was distinct from that in Arabidopsis. Pretreatment with $\mathrm{CK}$ at concentrations of 1 to $100 \mu \mathrm{M}$ had no significant effect on blast resistance (Fig. 5F), whereas higher doses of CK (110 to $460 \mu \mathrm{M})$ even increased blast disease incidence by 18 to 64\% (Matsumoto 1980). These results contrast with that observed in Arabidopsis, in which a low dose of CK $(0.1$ to $10 \mu \mathrm{M})$ treatment compromised while a high dose of $\mathrm{CK}(100 \mu \mathrm{M})$ increased resistance to $H$. arabidopsis Noco2 (Argueso et al. 2012). In tobacco, on the other hand, CK enhanced resistance to virulent $P$. syringae pv. tabaci by upregulating the synthesis of antimicrobial phytoalexins independent of SA (Grosskinsky et al. 2011). Overall, the assimilated data indicate that the mechanism by which $\mathrm{CK}$ regulate plant-pathogen interactions is quite complex and subjected to large variation across different pathosystems.

It has been recently shown that $\mathrm{CK}$ positively regulate resistance to $P$. syringae pv. tomato DC3000 and $H$. arabidopsis Noco 2 by modulating the SA signaling pathway in Arabidopsis (Argueso et al. 2012; Choi et al. 2010). Meanwhile, it is unlikely that CK directly modulate SA content or signaling in rice because i) SA levels do not alter (Silverman et al. 1995), despite the drastic accumulation of $\mathrm{CK}$ during blast infection (Table 1; Fig. 1); ii) CK treatment does not induce the expression of OsNPRI and WRKY45, nor does it further enhance SAinduced transcription levels (Jiang et al. 2010); and iii) CK treatment alone does not induce $P R$ gene expressions (Fig. 3). Moreover, in many dicots, such as Arabidopsis and tobacco, endogenous SA levels rapidly increase on pathogen infection, which leads to the induction of a battery of $P R$ genes (Vlot et al. 2009). In contrast, rice has constitutively high levels of endogenous SA that are approximately two orders of magnitude higher than the basal levels of SA in dicots, with pathogenic infections triggering no further increase in SA levels (Silverman et al. 1995). The mechanism by which the SA signaling pathway and the downstream defense genes are activated in response to pathogenic infections in rice is unknown. Some chemicals, termed plant activators (e.g., benzothiadiazole [BTH], which is a functional analog of SA), are known to induce disease resistance by potentiating SA-dependent defense gene expressions on pathogen infection, which causes minimum defense gene activation under unstressed conditions (Beckers and Conrath 2007). We previously isolated the rice WRKY45 gene, which plays a crucial role in BTH-induced blast resistance (Shimono 
et al. 2007). Overexpression of this gene enhances blast resistance, whereas $P R$ genes are not expressed in the absence of pathogen infection under greenhouse conditions (Shimono et al. 2007). These data suggest that additional, currently unknown signaling stimuli are required in addition to the SA signaling pathway for $P R$ gene inductions. Our findings regarding CK accumulation (Table 1; Fig. 1) and the synergistic interaction of CK with SA on $P R$ gene induction (Fig. 3) may provide a clue toward determining the mechanism of defense gene activation on pathogen infection in rice plants.

\section{MATERIALS AND METHODS}

\section{Plant materials and growth conditions.}

The Japonica rice Oryza sativa subsp. japonica 'Nipponbare' and transgenic lines for overexpression or RNAi-knockdown of rice WRKY45 (Shimono et al. 2007) or OsNPR1 (Sugano et al. 2010), OsRR6::GUS (discussed below), and GVG-CKX2 (discussed below) were grown in a greenhouse in soil (Bonsol Number 2; Sumitomo Chemical Corp., Tokyo) at $28^{\circ} \mathrm{C}$ in the day $(14 \mathrm{~h})$ and $23^{\circ} \mathrm{C}$ in the night $(10 \mathrm{~h})$. The relative humidity in the greenhouse was approximately $70 \%$.

\section{DNA construction and plant transformation.}

To construct OsRR6 promoter::GUS DNA (OsRR6::GUS), the genomic region containing putative promoter regions of OsRR6 (2.0 kb from the translational initiation codon) were amplified by PCR with genomic DNA and specific primers $\left(5^{\prime} \rightarrow 3^{\prime}\right)$ TAAGCTTACCACAGCAGCAGCTAGCAAATC and TGTCGACTGATCAACTCAAAATCCAACC. The resulting PCR product was cloned into pCR-Blunt-TOPO (Invitrogen, Carlsbad, CA, U.S.A.) and verified by complete sequencing. The verified fragments were introduced into the upstream region of the open reading frame of the GUS gene that is inserted into the HindIII-SalI site of the pCAMBIA1390 vector (CAMBIA, Canberra, Australia).

To construct $G V G: \because C K X 2$ DNA, rice gene $C K X 2$ (Ashikari et al. 2005) was PCR amplified using primers $\left(5^{\prime} \rightarrow 3^{\prime}\right)$ ATACT AGTCATGCTCAACTGCTTCGTCA and ATCTCGAGACGC ATGTAGATATGCATTTGCATC. The resulting PCR product was cloned into the SpeI-XhoI site of the dexamethasoneinducible vector PTA7001 (Aoyama and Chua 1997) and confirmed by sequencing.

Transgenic rice plants were generated by the Agrobacteriummediated method (Hirose et al. 2005) using the Agrobacterium tumefaciens strain EHA105.

\section{Chemical treatments.}

All stock solutions, except for brassinolide (BR), were prepared at a concentration of $100 \mathrm{mM}$ as described previously (Jiang et al. 2008). BR was prepared at $20 \mathrm{mM}$ concentration. IAA (Sigma-Aldrich, St. Louis), gibberellin A3 (GA $)$ (Wako, Osaka, Japan), ABA (( \pm$)$-cis-trans; Sigma-Aldrich), methyl jasmonate (Wako), and BR (Wako) were dissolved in ethanol. Kinetin (Sigma-Aldrich), IP (Sigma-Aldrich), and BTH $S$-methyl ester (Wako) were dissolved in dimethyl sulfoxide; and 1aminocyclopropane-1-carboxylic acid (Sigma-Aldrich) and sodium salicylate (Nacalai Tesque, Tokyo, Japan) in $\mathrm{H}_{2} \mathrm{O}$. The solvents did not exceed a final concentration of $0.1 \%$ in the solutions used for plant treatments, and had no effect on the expression of rice genes examined in this work.

Leaf blades from rice plants at the four-leaf stage were cut into segments of approximately $0.5 \mathrm{~cm}$ in length and submerged in a solution containing chemicals prepared in $0.01 \%$ Silwet L-77. The leaf segments were then incubated under light $\left(60 \mu \mathrm{mol} \mathrm{m} \mathrm{m}^{-2} \mathrm{~s}^{-1}\right)$ for 6 to $8 \mathrm{~h}$ at $30^{\circ} \mathrm{C}$ in a growth chamber. For plant treatment, chemicals prepared in $0.02 \%$ Lelwet
L-77 were sprayed onto rice seedlings as described previously (Shimono et al. 2007).

\section{Pathogen culture and inoculations.}

Culture and inoculations of the blast fungus M. oryzae (race 007.0) were carried out essentially as described previously (Jiang et al. 2009), with slight modifications. Briefly, the fungus was grown on an oatmeal agar medium (oatmeal at 30 $\mathrm{g} /$ liter, sucrose at $5 \mathrm{~g} / \mathrm{liter}$, and agar at $16 \mathrm{~g} /$ liter) at $26^{\circ} \mathrm{C}$ for 10 to 12 days. Conidia formation was induced by irradiation under continuous black-blue light (FL15BLB; Toshiba, Osaka, Japan) at $24^{\circ} \mathrm{C}$ for 2 to 4 days. The conidia were suspended in $0.02 \%$ Silwet L-77 at a density of 1 to $2 \times 10^{5}$ conidia per milliliter and were sprayed onto rice plants at the four-leaf stage. After incubation in a dew chamber at $24^{\circ} \mathrm{C}$ for $24 \mathrm{~h}$, the rice plants were moved back to the greenhouse. Disease development was evaluated either by counting the number of blast lesions per $10-\mathrm{cm}$ middle region of leaf blade or by determining M. oryzae genomic 28S rDNA (Qi and Yang 2002) by qRT-PCR at 6 days after inoculation. Approximately 10 to 15 plants were used for each disease assay.

\section{RNA analyses.}

Total RNA was isolated from leaf blades of the fourth leaf of rice seedlings using the Trizol reagent (Invitrogen) and reverse-transcribed by using ReverTra Ace (TOYOBO, Osaka, Japan) according to the manufacturer's protocol. qRT-PCR was run on a Thermal Cycler Dice TP800 system (Takara Bio, Tokyo) using SYBR premix Ex Taq mixture (Takara Bio) as previously described (Shimono et al. 2007). Primer sequences used for qRT-PCR are listed in Supplementary Table S1.

\section{Measurement of hormone contents.}

Rice leaf blades were collected in liquid nitrogen at various time points after blast inoculation. The hyphae, conidia, and culture filtrates of fungus $M$. oryzae (races 007.0 and 102.0) and culture medium as control were prepared as previously described (Jiang et al. 2010). The hormone determination was carried out as described previously with an ultra-performance liquid chromatography (UPLC) tandem mass spectrometry (AQITY UPLC System/Quattro Ultima Pt; Waters, Milford, MA, U.S.A.) with an ODS column (AQUITY UPLC BEH C18, $1.7 \mu \mathrm{m}, 2.1$ by $100 \mathrm{~mm}$; Waters) (Kojima et al. 2009).

\section{GUS staining and microscopic observation.}

The GUS staining was carried out according to Ruzin (1999). For histochemical analysis, OsRR6::GUS transgenic rice plants were inoculated with blast fungus race 007.0. The leaf blades at 2 days postinoculation were stained for GUS activity. Sections were viewed under a stereo microscope (Leica MZFL III) and photomicrographs were obtained using a digital CCD camera (Sony DXC-9000, Tokyo).

\section{ACKNOWLEDGMENTS}

This work was supported by a Grant-in-Aid for Scientific Research (number 22570057) from the Ministry of Education, Culture, Sports, Science and Technology, Japan and a grant from the Japanese Ministry of Agriculture, Forestry and Fisheries (Genomics for Agricultural Innovation, GMA0001 and PMI0008). C.-J. Jiang designed the research and wrote the article, M. Shimono and S. Sugano produced the transgenic rice plants, M. Kojima and H. Sakakibara analyzed hormone levels, X. Liu and H. Inoue analyzed gene expressions, and $\mathrm{H}$. Takatsuji analyzed and interpreted the data.

\section{LITERATURE CITED}

Agrawal, G. K., Rakwal, R., and Jwa, N. S. 2000. Rice (Oryza sativa L.) $O s P R 1 b$ gene is phytohormonally regulated in close interaction with 
light signals. Biochem. Biophys. Res. Commun. 278:290-298.

Aoyama, T., and Chua, N. H. 1997. A glucocorticoid-mediated transcriptional induction system in transgenic plants. Plant J. 11:605-612.

Argueso, C. T., Raines, T., and Kieber, J. J. 2010. Cytokinin signaling and transcriptional networks. Curr. Opin. Plant Biol. 13:533-539.

Argueso, C. T., Ferreira, F. J., Epple, P., Ton, J., Hutchison, C. E., Schaller, G. E., Dangl, J. L., and Kieber, J. J. 2012. Two-component elements mediate interactions between cytokinin and salicylic acid in plant immunity. PLoS Genet. 8:1-13. Published online.

Ashby, A. M. 2000. Biotrophy and the cytokinin conundrum. Physiol. Mol. Plant Pathol. 57:147-158.

Ashikari, M., Sakakibara, H., Lin, S., Yamamoto, T., Takashi, T., Nishimura, A., Angeles, E.R., Qian, Q., Kitano, H., and Matsuoka, M. 2005. Cytokinin oxidase regulates rice grain production. Science 309:741-745.

Asselbergh, B., De Vleesschauwer, D., and Hofte, M. 2008. Global switches and fine-tuning-ABA modulates plant pathogen defense. Mol. Plant-Microbe Interact. 21:709-719.

Beckers, G. J., and Conrath, U. 2007. Priming for stress resistance: from the lab to the field. Curr. Opin. Plant Biol. 10:425-431.

Behr, M., Humbeck, K., Hause, G., Deising, H. B., and Wirsel, S. G. 2010 The hemibiotroph Colletotrichum graminicola locally induces photosynthetically active green islands but globally accelerates senescence on aging maize leaves. Mol. Plant-Microbe Interact. 23:879-892.

Behr, M., Motyka, V., Weihmann, F., Malbeck, J., Deising, H. B., and Wirsel, S.G. 2012. Remodeling of cytokinin metabolism at infection sites of Colletotrichum graminicola on maize leaves. Mol. PlantMicrobe Interact. 25:1073-1082.

Berruyer, R., Poussier, S., Kankanala, P., Mosquera, G., and Valent, B. 2006. Quantitative and qualitative influence of inoculation methods on in planta growth of rice blast fungus. Phytopathology 96:346-355.

Choi, J., Huh, S. U., Kojima, M., Sakakibara, H., Paek, K. H., and Hwang, I. 2010. The cytokinin-activated transcription factor ARR2 promotes plant immunity via TGA3/NPR1-dependent salicylic acid signaling in Arabidopsis. Dev. Cell 19:284-295.

Choi, J., Choi, D., Lee, S., Ryu, C. M., and Hwang, I. 2011. Cytokinins and plant immunity: old foes or new friends? Trends Plant Sci. 16:388394

Choi, J., Lee, J., Kim, K., Cho, M., Ryu, H., An, G., and Hwang, I. 2012. Functional identification of OsHk6 as a homotypic cytokinin receptor in rice with preferential affinity for iP. Plant Cell Physiol. 53:13341343

Ding, X., Cao, Y., Huang, L., Zhao, J., Xu, C., Li, X., and Wang, S. 2008. Activation of the indole-3-acetic acid-amido synthetase GH3-8 suppresses expansin expression and promotes salicylate- and jasmonateindependent basal immunity in rice. Plant Cell 20:228-240.

Domingo, C., Andres, F., Tharreau, D., Iglesias, D. J., and Talon, M. 2009. Constitutive expression of OsGH3.1 reduces auxin content and enhances defense response and resistance to a fungal pathogen in rice. Mol. Plant-Microbe Interact. 22:201-210.

Galichet, A., Hoyerova, K., Kaminek, M., and Gruissem, W. 2008. Farnesylation directs AtIPT3 subcellular localization and modulates cytokinin biosynthesis in Arabidopsis. Plant Physiol. 146:1155-1164.

Grant, M. R., and Jones, J. D. 2009. Hormone (dis)harmony moulds plant health and disease. Science 324:750-752.

Grosskinsky, D. K., Naseem, M., Abdelmohsen, U. R., Plickert, N., Engelke, T., Griebel, T., Zeier, J., Novak, O., Strnad, M., Pfeifhofer, H., van der Graaff, E., Simon, U., and Roitsch, T. 2011. Cytokinins mediate resistance against Pseudomonas syringae in tobacco through increased antimicrobial phytoalexin synthesis independent of salicylic acid signaling. Plant Physiol. 157:815-830.

Ha, S., Vankova, R., Yamaguchi-Shinozaki, K., Shinozaki, K., and Tran, L. S. 2012. Cytokinins: metabolism and function in plant adaptation to environmental stresses. Trends Plant Sci. 17:172-179.

Hasegawa, M., Mitsuhara, I., Seo, S., Imai, T., Koga, J., Okada, K., Yamane, H., and Ohashi, Y. 2010. Phytoalexin accumulation in the interaction between rice and the blast fungus. Mol. Plant-Microbe Interact. 23:1000-1011.

Hirose, N., Makita, N., Yamaya, T., and Sakakibara, H. 2005. Functional characterization and expression analysis of a gene, OsENT2, encoding an equilibrative nucleoside transporter in rice suggest a function in cytokinin transport. Plant Physiol. 138:196-206.

Hirose, N., Makita, N., Kojima, M., Kamada-Nobusada, T., and Sakakibara, H. 2007. Overexpression of a type-A response regulator alters rice morphology and cytokinin metabolism. Plant Cell Physiol. 48:523539

Ito, Y., and Kurata, N. 2006. Identification and characterization of cytokinin-signalling gene families in rice. Gene 382:57-65.

Jain, M., Tyagi, A. K., and Khurana, J. P. 2006. Molecular characterization and differential expression of cytokinin-responsive type-A response regulators in rice (Oryza sativa). BMC Plant Biol. 6:1.

Jameson, P. 2000. Cytokinins and auxins in plant-pathogen interactionsAn overview. Plant Growth Regul. 32:369-380.

Jiang, C. J., Aono, M., Tamaoki, M., Maeda, S., Sugano, S., Mori, M., and Takatsuji, H. 2008. SAZ, a new SUPERMAN-like protein, negatively regulates a subset of ABA-responsive genes in Arabidopsis. Mol. Genet. Genomics 279:183-192.

Jiang, C. J., Shimono, M., Maeda, S., Inoue, H., Mori, M., Hasegawa, M. Sugano, S., and Takatsuji, H. 2009. Suppression of the rice fatty-acid desaturase gene OsSSI2 enhances resistance to blast and leaf blight diseases in rice. Mol. Plant-Microbe Interact. 22:820-829.

Jiang, C. J., Shimono, M., Sugano, S., Kojima, M., Yazawa, K., Yoshida, R., Inoue, H., Hayashi, N., Sakakibara, H., and Takatsuji, H. 2010. Abscisic acid interacts antagonistically with salicylic acid signaling pathway in rice-Magnaporthe grisea interaction. Mol. Plant-Microbe Interact. 23:791-798.

Kachroo, A., and Kachroo, P. 2007. Salicylic acid-, jasmonic acid- and ethylene-mediated regulation of plant defense signaling. Genet. Eng. (N.Y.) 28:55-83.

Kankanala, P., Czymmek, K., and Valent, B. 2007. Roles for rice membrane dynamics and plasmodesmata during biotrophic invasion by the blast fungus. Plant Cell 19:706-724.

Ko, K. W., Okada, K., Koga, J., Jikumaru, Y., Nojiri, H., and Yamane, H. 2010. Effects of cytokinin on production of diterpenoid phytoalexins in rice. J. Pestic. Sci. 35:412-418.

Kojima, M., Kamada-Nobusada, T., Komatsu, H., Takei, K., Kuroha, T. Mizutani, M., Ashikari, M., Ueguchi-Tanaka, M., Matsuoka, M., Suzuki, K., and Sakakibara, H. 2009. Highly sensitive and highthroughput analysis of plant hormones using MS-probe modification and liquid chromatography-tandem mass spectrometry: an application for hormone profiling in Oryza sativa. Plant Cell Physiol. 50:12011214.

Kudo, T., Kiba, T., and Sakakibara, H. 2010. Metabolism and long-distance translocation of cytokinins. J. Integr. Plant Biol. 52:53-60.

Loake, G., and Grant, M. 2007. Salicylic acid in plant defence-The players and protagonists. Curr. Opin. Plant Biol. 10:466-472.

Lopez, M. A., Bannenberg, G., and Castresana, C. 2008. Controlling hormone signaling is a plant and pathogen challenge for growth and survival. Curr. Opin. Plant Biol. 11:420-427.

Matsumoto, K. 1980. On the relationship between plant hormones and rice blast resistance. Ann. Phytopathol. Soc. Jpn. 46:307-314.

Midoh, N., and Iwata, M. 1996. Cloning and characterization of a probenazole-inducible gene for an intracellular pathogenesis-related protein in rice. Plant Cell Physiol. 37:9-18.

Peleg, Z., Reguera, M., Tumimbang, E., Walia, H., and Blumwald, E. 2011. Cytokinin-mediated source/sink modifications improve drought tolerance and increase grain yield in rice under water-stress. Plant Biotechnol. J. 9:747-758.

Perfect, S. E., Hughes, H. B., O'Connell, R. J., and Green, J. R. 1999. Colletotrichum: a model genus for studies on pathology and fungalplant interactions. Fungal Genet. Biol. 27:186-198.

Perilli, S., Moubayidin, L., and Sabatini, S. 2010. The molecular basis of cytokinin function. Curr. Opin. Plant Biol. 13:21-26.

Qi, M., and Yang, Y. 2002. Quantification of Magnaporthe grisea during infection of rice Plants using real-time polymerase chain reaction and northern blot/phosphoimaging analyses. Phytopathology 92:870-876.

Ribot, C., Hirsch, J., Balzergue, S., Tharreau, D., Notteghem, J. L. Lebrun, M. H., and Morel, J. B. 2008. Susceptibility of rice to the blast fungus, Magnaporthe grisea. J. Plant Physiol. 165:114-124.

Robert-Seilaniantz, A., Navarro, L., Bari, R., and Jones, J. D. 2007. Pathological hormone imbalances. Curr. Opin. Plant Biol. 10:372-379.

Robert-Seilaniantz, A., Grant, M., and Jones, J. D. 2011. Hormone crosstalk in plant disease and defense: more than just jasmonate-salicylate antagonism. Annu. Rev. Phytopathol. 49:317-343.

Ruzin, S. E. 1999. Plant Microtechnique and Microscopy. Oxford University Press, Oxford.

Sakakibara, H. 2006. Cytokinins: activity, biosynthesis, and translocation. Annu. Rev. Plant Biol. 57:431-449.

Sakamoto, T., Sakakibara, H., Kojima, M., Yamamoto, Y., Nagasaki, H., Inukai, Y., Sato, Y., and Matsuoka, M. 2006. Ectopic expression of KNOTTED1-like homeobox protein induces expression of cytokinin biosynthesis genes in rice. Plant Physiol. 142:54-62.

Sano, H., Seo, S., Orudgev, E., Youssefian, S., and Ishizuka, K. 1994. Expression of the gene for a small GTP binding protein in transgenic tobacco elevates endogenous cytokinin levels, abnormally induces salicylic acid in response to wounding, and increases resistance to tobacco mosaic virus infection. Proc. Natl. Acad. Sci. U.S.A. 91:1055610560 .

Shimono, M., Sugano, S., Nakayama, A., Jiang, C. J., Ono, K., Toki, S., 
and Takatsuji, H. 2007. Rice WRKY45 plays a crucial role in benzothiadiazole-inducible blast resistance. Plant Cell 19:2064-2076.

Siemens, J., Keller, I., Sarx, J., Kunz, S., Schuller, A., Nagel, W., Schmulling, T., Parniske, M., and Ludwig-Muller, J. 2006. Transcriptome analysis of Arabidopsis clubroots indicate a key role for cytokinins in disease development. Mol. Plant-Microbe Interact. 19:480-494.

Silverman, P., Seskar, M., Kanter, D., Schweizer, P., Metraux, J. P., and Raskin, I. 1995. Salicylic acid in rice: biosynthesis, conjugation, and possible role. Plant Physiol. 108:633-639.

Stes, E., Vandeputte, O. M., El Jaziri, M., Holsters, M., and Vereecke, D. 2011. A successful bacterial coup d'etat: how Rhodococcus fascians redirects plant development. Annu. Rev. Phytopathol. 49:69-86.

Sugano, S., Jiang, C. J., Miyazawa, S., Masumoto, C., Yazawa, K. Hayashi, N., Shimono, M., Nakayama, A., Miyao, M., and Takatsuji, H. 2010. Role of OsNPR1 in rice defense program as revealed by genomewide expression analysis. Plant Mol. Biol. 74:549-562.
Swartzberg, D., Kirshner, B., Rav-David, D., Elad, Y., and Granot, D. 2008. Botrytis cinerea induces senescence and is inhibited by autoregulated expression of the IPT gen. Eur. J. Plant Pathol. 120:289-297.

Talbot, N. J., Ebbole, D. J., and Hamer, J. E. 1993. Identification and characterization of $M P G 1$, a gene involved in pathogenicity from the rice blast fungus Magnaporthe grisea. Plant Cell 5:1575-1590.

Vlot, A. C., Dempsey, D. A., and Klessig, D. F. 2009. Salicylic acid, a multifaceted hormone to combat disease. Annu. Rev. Phytopathol. 47:177-206.

Walters, D. R., and McRoberts, N. 2006. Plants and biotrophs: a pivotal role for cytokinins? Trends Plant Sci. 11:581-586.

Yazawa, K., Jiang, C. J., Kojima, M., Sakakibara, H., and Takatsuji, H. 2012. Reduction of abscisic acid levels or inhibition of abscisic acid signaling in rice during the early phase of Magnaporthe oryzae infection decreases its susceptibility to the fungus. Physiol. Mol. Plant Pathol. 78:1-7. 